\title{
Optimization of shifting strategy for a dual clutch transmission through mathematical model
}

\author{
Norbert Bagameri ${ }^{1}$, Bogdan Varga $^{1}$, Aron Csato ${ }^{1}$ and Dan Moldovanu ${ }^{1, *}$ \\ ${ }^{1}$ Technical University of Cluj-Napoca, Automotive and Transport Department, 400641 Cluj-Napoca, Romania
}

\begin{abstract}
European Union legislation sets mandatory emission and fuel consumption reduction targets for new vehicles and therefore economy remains a key challenge for automotive engineers. Optimizing the transmission shift schedule for an automatic transmission with double clutch is an important part of improving fuel economy. Using simulation and optimization algorithms can ensure that a truly optimal shift schedule is implemented. The main objective of this paper focuses on study the performance of a vehicle with a dual clutch transmission (DCT), with special emphasis on optimizing the shifting schedule using optimization algorithms. In order to study and simulate the shifting strategy the dynamic model is developed based on an existing model in MATLAB Simulink software. In the first phase of the paper a detailed description of the DCT technology, its main functional components and dynamic characteristics are explored. In the second phase model development and simulation concerns the major part of this work, to find the optimal shift schedule for different engine speed and engine torque as it accelerates through the transmission gears by engaging the dual clutches. Finally, the simulation results were evaluated and presented which successfully demonstrate the performance improvement of a dual clutch transmission with an optimized shifting strategy.
\end{abstract}

\section{Introduction}

The function of a vehicle transmission is to adapt the traction available from the drive unit to suit the vehicle, the surface, the driver and the environment. It has a decisive effect on the reliability, fuel consumption, ease of use, road safety and transportation performance. The optimization of the transmission can significantly increase the performance of the vehicle efficiency and fuel economy [1]. One of the main challenges in the automotive industry is to improve mechanical efficiency of the drivetrain and in the same time to ensure high comfort level at the board of the vehicle. [2]. The Dual Clutch Transmission is a new type of automatic transmission device in the field of automotive automatic transmission. It realizes power transmission and shifting without power interruption through respective connection between the two-transmission shaft and two clutches, which improve better fuel economy. Shift control is the key technology of DCT vehicles, and an important index to measure the driving comfort of vehicles, therefore, to carry out targeted shift analysis and control of the DCT seems quite necessary [3, 4]. A dual clutch transmission is quite similar to the conventional automatic, the main difference being the double clutch structure compared to the single automatic clutch used in automatics. Automatics make the use of a torque converter to transfer engine torque from the engine to the transmission, DCTs on the other hand don't require torque converters [5].

\section{Structure and principle of DCT}

In the following paragraph, a short description of the dual clutch transmission is presented.. When shifting between gear ratios, the transmission control unit (TCU) should synchronize the engagement of the on-coming clutch and the disengagement of the off-going clutch, this process is called clutch-to-clutch shift control [6]. Under dynamic driving conditions, the transmission is required to shift in order to match the engine power with the changing drive power. Furthermore, a gearshift decision is expected to be consistent such that the vehicle can remain in the next gear for a period of time without deteriorating the acceleration capability. Therefore, an optimal conversion of the engine power plays a key role in improving the fuel economy and driveability. Similar with the traditional manual gearbox structure it includes cylindrical gear sets and synchroniser gear set. Fig. 1 shows the layout of the DCT mounted on a front wheel drive vehicle, with transversal internal combustion engine and gearbox. All the gears of the transmission have an identification number, which is used to specify the gear transmission ratios.

\footnotetext{
* Corresponding author: dan.moldovanu@auto.utcluj.ro
} 


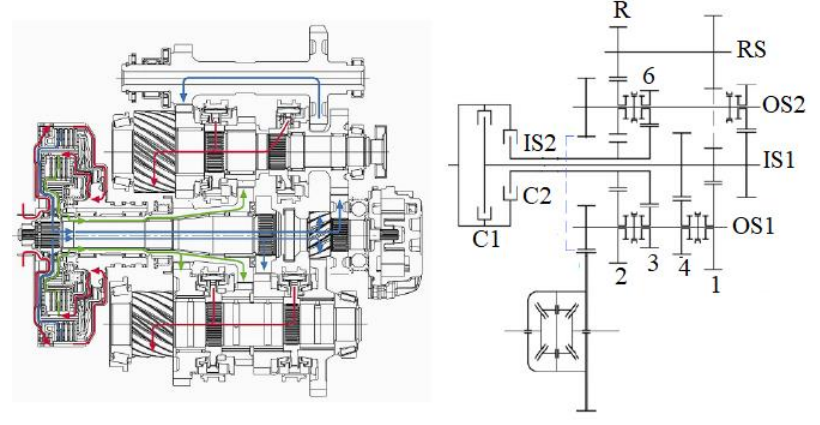

Fig. 1. Cross section and layout of the DCT [7].

The two clutches, that give the name to the transmission, can be single or multiple disk, dry or wet type, depending mainly on the amount of power they have to transfer and they are mounted between the engine flywheel and one of the two coaxial input shafts of the transmission, one for odd gears and the other for even gears. Gearshift and engagement of clutches are electronically managed by a control unit, which drives the mechatronic assembly integrated in the gearbox. The gear shift from first to second gear is automatically actuated by the transmission control unit (TCU) in a short time thanks to the concurrent disengagement of the first clutch and engagement of the second clutch, thus eliminating the dead times due to the traditional synchronising phase. Hence, this clutch-to-clutch shift allows not only to accomplish the gearshift, but also to have a continuous torque transfer to the wheels (power-shift) during the gear change process. Obviously, a similar procedure is repeated when the engagement of the second gear is completed: the system gets ready to the following gear shift inserting the synchroniser corresponding to the next gear [4], [7]. A distinctive characteristic of the DCT is represented by the fact that during normal operating conditions the dual clutch gearbox has two different gear ratios engaged at the same time: the TCU must avoid the concurrent lock of the two clutches in order to prevent damages to the gearbox [4].

\section{Simulation Software}

In order to analyse the dynamic characteristics of the vehicle and to study performance, a computer simulation model of the vehicle system, using Matlab Simulink software package has been developed. The software Simulink and Matlab are available from the Mathworks, which provides a graphical interface for building models as block diagrams. Matlab Simulink provides a platform to simulate the performance of the optimal shifting controller by running the transmission model in a certain driving cycle. In this model, the Transmission Controller block mainly has several inputs and outputs; the main inputs in this model are the vehicle speed, the internal combustion engine speed, the clutch slipping sensor signal, and the command speed, and the main outputs are the gear, pressure and torque demanded, which are used to drive the transmission.

With the definition of system notion and the development of a mathematical device to realize the connection between input parameters, output parameters and operating parameters of the system modeling process began to be increasingly used in all areas related to human activities. Expression of relations between input and output values of the systems is achieved by using mathematical functions. The simulation was performed using Matlab Simulink software package in order to obtain the optimal shifting schedule of the dual clutch transmission for fuel economy of the vehicle model.

\section{Vehicle subsystems}

The powertrain consists of functional components in the form of blocks such as vehicle dynamics, engine model, input and output shafts, transmission, transmission controller, wheels and tires. The characteristic curve of an internal combustion engine defines the torque supplied as function of engine speed and throttle valve opening. The transmission subsystem is the focal point of the powertrain model, this is dual clutch transmission with a six forward gear sets and one reverse gear set. The dual clutch transmission is utilize clutches to transfer torque at various speed ratios through the transmission. The synchronizers cause discontinuities in the passage from synchronization to engaged gear: the interaction between elements, initially due to friction is then realize by the contact of the dog-teeth. Stationary idle, transition to motion and interruption of the power flow are all made possible by the clutch. The clutch slips to compensate for the difference in the rotational speeds of engine and drivetrain when the vehicle is set in motion. When a change in operation conditions makes it necessary to change gears clutch disengages the engine from the drivetrain for the duration of procedure. The clutch is controlled by the driver via the cockpit. The maximum transferable torque is the maximum torque the clutch can transfer between the drive and the power take-off side [6].

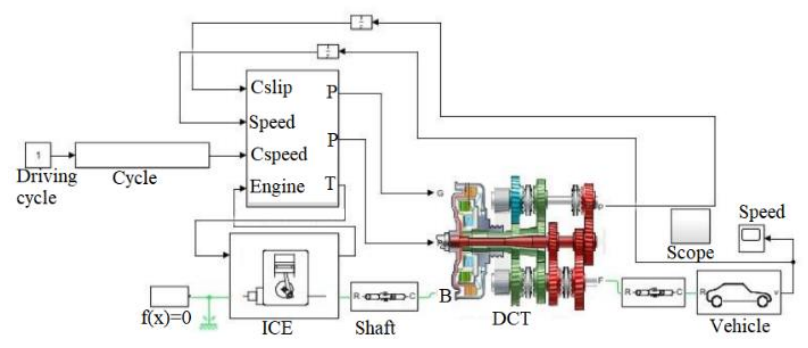

Fig. 2. Vehicle model

\section{Shifting strategy}

For conventional vehicles equipped with discrete ratio transmissions, e.g. automatic, dual clutch, automated (manual) transmissions, etc., a decision on selecting a different gear leads to a sudden change of the engine operating point due to a stepped change of the transmission ratio. With highly nonlinear characteristics of the engine fuel consumption and maximum torque, a change of the engine operating point due to a gearshift can result in a deficient fuel operation state, and/or a low driveability condition for the vehicle thereafter. Hence, an 
optimal gear shift strategy for a discrete ratio transmission plays an important role in achieving a high performance for the vehicle [8-9]. The shift process of dual clutch consists of two stages: the torque stage is the conversion process of torque, that is, the separation of the torque from the clutch converts to engaging to it; the inertial stage is the speed synchronization process, namely, the engine speed synchronizes with the target gear speed. When control the throttle to up shift the gear, first, entering the torque stage, and then enters the inertia stage, in the downshift process, it is the opposite. The second stage is the synchronous stage in this stage, the clutch is stable, the speeds of the master and the side driven plates are equal. When the transfer torque is less than the capacity of the clutch torque, the clutch torque is equal to the current transmission torque, while when the transfer torque is bigger than the capacity of the clutch torque, the clutch torque is equal to the maximum transfer torque of the clutch [8].

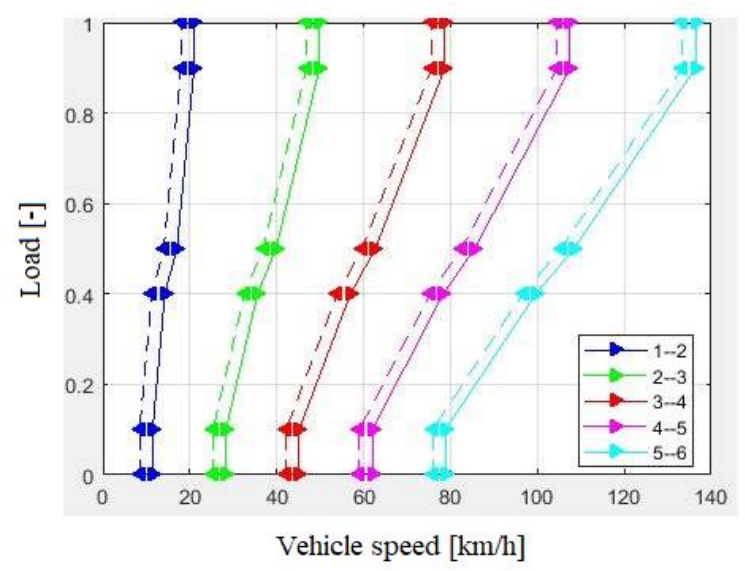

Fig. 3. Shifting strategy

In a real world application, the dual clutch transmission controller has several gear shifting strategy's for different driving conditions. According to the situation the TCU decides which strategy is using.

\section{Simulation and results}

In this chapter, an optimal control strategy for the gear shifting schedule of the automatic transmission is developed depending on the proper application of the decision-making algorithm for best fuel economy. The shifting schedule is optimized for FTP75 cycle, with helping of optimization solvers. At first parameters of the shifting schedule is optimized. Two parameters of the shifting schedule are identified the distance between the 0 load shifting lines and the distance between full load shifting lines. In the first and second optimization only the shifting schedule parameters are optimized with two different solvers. The third optimization solver was using 30 parameters to optimize the shifting schedule.

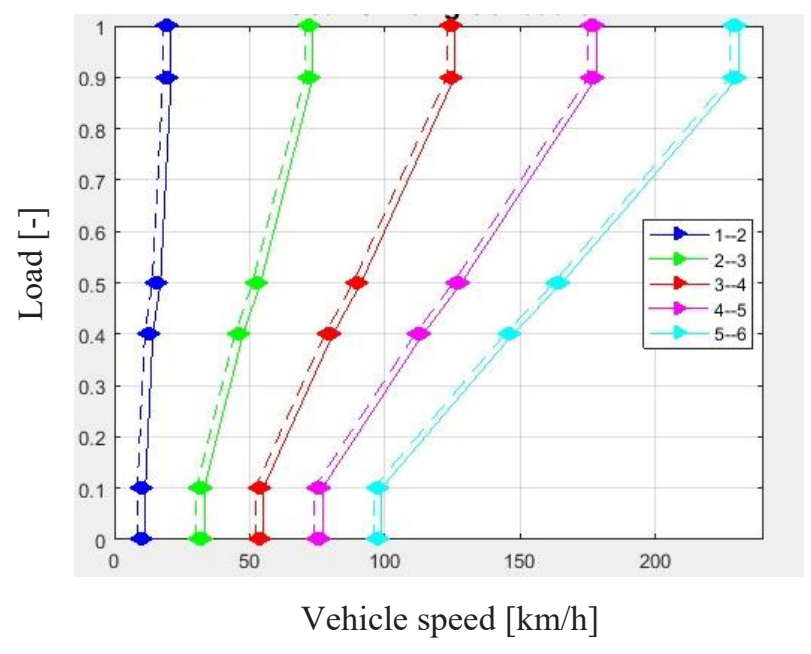

Fig. 4. First optimization shifting schedule

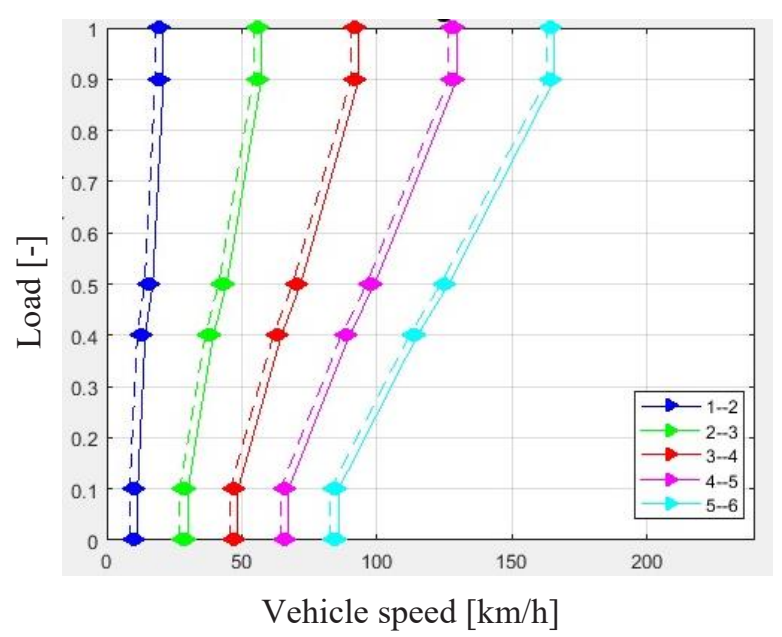

Fig. 5. Second optimization shifting schedule

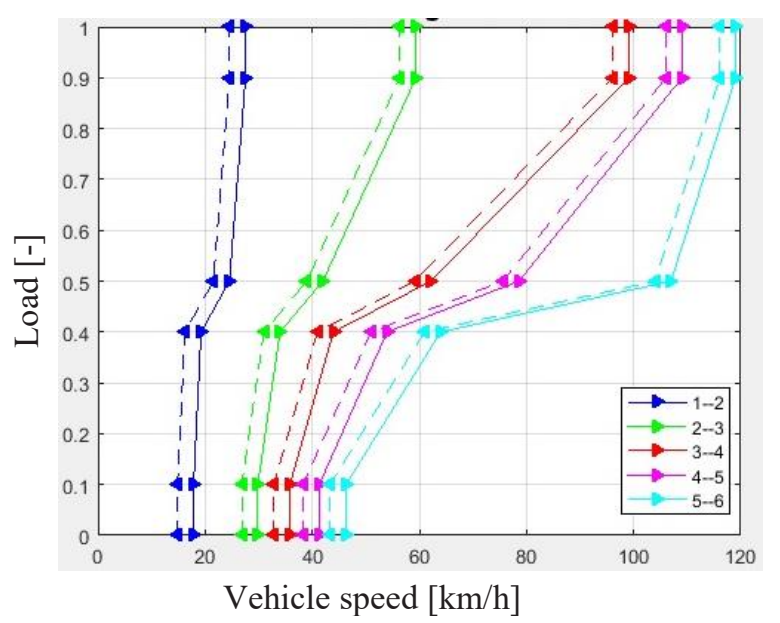

Fig. 6. Third optimization shifting schedule

After the integrated control analysis of the DCT shift and the formulation of control strategy, on the basis of MATLAB Simulink (Mathworks) simulation platform, simulation model and simulation analysis has been made on the shifting process for the six speed dual clutch transmission. An optimal gear shift strategy, allowing the engine to operate in the region closely to maximum torque 
of the engine in every gear because this offers minimal consumption.

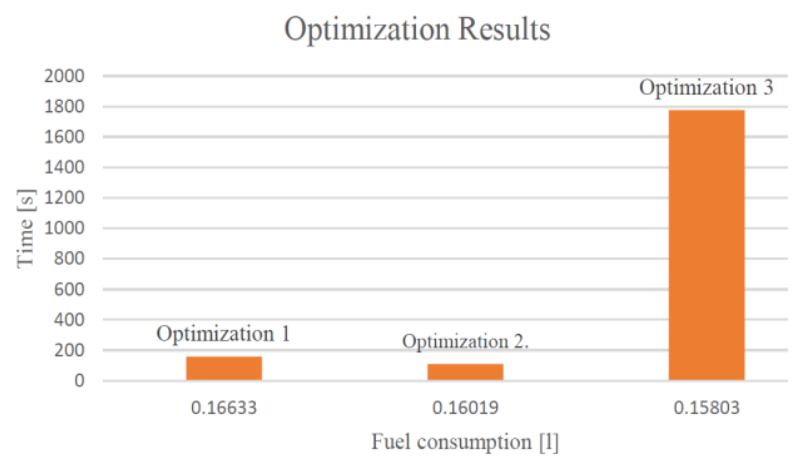

Fig. 7. Optimization results

The optimization process take several iteration, in the 3rd case is more than 10000 iteration to find the lowest fuel consumption. Once we have a complete model, we can run the simulation for any shift schedule that we want to test. Improved fuel economy is probably the most significant benefit of using a dual clutch transmission due to the fact that during gearshifts power delivery from the engine to the transmission is uninterrupted and therefore fuel efficiency increases dramatically.

\section{Conclusion}

The control strategy of the engine and clutch and the comprehensive shift control strategy are made in the DCT shifting process. Based on the Matlab Simulink software simulation platform, simulation model of vehicle shifting has been developed, and the simulation results validates affectivity of the shift model, and it also indicates the good control effect of the formulated shift control strategy. Modelling and simulation approach of technical and functional characteristics that influence the powertrain is widely presented in this paper. The potential for optimization the shifting strategy for increasing the overall efficiency of the vehicle strongly depends on the driving cycle that it is applied to, disturbances from traffic and driving behaviour. In Matlab Simulink model are simulated a powertrain and a drive train. For optimizing the shifting schedule the model is running on FTP 75 cycle and the solvers are minimizing the fuel consumption by trying different shifting schedules. Optimization process can be used for different engines that uses the same DCT transmission. These optimization solvers are based on finding minimum values so they are faster than brute force optimization. The fuel-optimal gearshift strategy, proposed on Fig. 6, yields an early and frequent upshift pattern for the transmission. It renders the engine, on average, to operate at a lower speed and high torque region, and hence improving the fuel economy. For facing the high requirements of the new emission regulations every optimization have to be considered, so a correct optimization for gear schedule could have a high impact on the emissions.

\section{References}

1. W. Sarawoot "Modeling and estimation for stepped automatic transmission with clutch-to-clutch shift technology" Dissertation Thesis, Ohio State University, 2004.

2. A. Faraz, "Modelling and simulation of a vehicle with a controlled dual clutch transmission" University of Bradford, 2015.

3. G. Enrico, A. Vigliani, M. Velardocchia, "Dynamic and kinematic model of dual clutch transmission," Mechanism and machine theory 794-805, 2011.

4. N. Chunsheng, L. Tongli, J. Zhang, "Gearshift control for dry dualclutch transmissions” Issue 11, Vol.8, pp. 1177-1186, 2009.

5. X. Xiaohui "Shifting Control and Analysis of Dual Clutch Transmission of Automobile." International Journal of Control and Automation Vol.8, No.12, pp.241-252, 2015.

6. N. Dac, "Gear shift strategies for Automotive Trasnsmission" Eindhoven University of Technology, ISBN 978-90-386-3222-3, 2012.

7. N. Chunsheng, L. Tongli, J. Zhang, "Gearshift control for dry dualclutch transmissions" Issue 11, Vol.8, pp. 1177-1186, 2009.

8. Y. Wang, "Optimal Gear Shifting Strategy for a Seven-speed Automatic Transmission Used on a Hydraulic Hybrid Vehicle" Dissertation Thesis, University of Toledo, 2012.

9. F. Meng, G. Tao, H. Chen, "Smooth shift control of an automatic transmission for heavy-duty vehicles," Journal Neurocomputing, 159 , pp.197-206, 2015 